\title{
Online-Plattform «Qualitätsinitiativen SAQM» - neu konzipiert
}

\author{
Nadja Jennia, Esther Kraft ${ }^{\text {b }}$ \\ ${ }^{a}$ M. Sc., Wissenschaftliche Mitarbeiterin FMH/SIWF; ${ }^{b}$ Lic. rer. oec., Abteilungsleiterin DDO
}

\begin{abstract}
Ziel der Qualitätsarbeit ist es, auf Basis vorhandener Ressourcen, die Qualität der Patientenversorgung zu optimieren und Massnahmen der Qualitätssicherung zu implementieren. Qualität im Gesundheitswesen ist eine herausfordernde und sich immer im Wandel befindliche Materie. Entsprechend hat sich die FMH zum Ziel gesetzt, die bestehende Online-Plattform der Qualitätsinitiativen neu zu konzipieren, zu erweitern und auszubauen. Aktivitäten im Qualitätsmanagement verhelfen nicht nur zur Verbesserung der Patientensicherheit, sondern sie nützen dem gesamten Gesundheitswesen.
\end{abstract}

Von allen Seiten ertönt der Ruf nach mehr Transparenz in der medizinischen Qualität. Die Qualität auf einem hohen Niveau zu halten, verlangt von der Ärzteschaft und allen weiteren Akteuren im Gesundheitswesen einen grossen Einsatz. Entsprechend zahlreich sind die Initiativen zur Qualitätssicherung. Seit 2007 dokumentiert die FMH/SAQM Qualitätsaktivitäten auf ihrer Website (www.fmh.ch $\rightarrow$ Qualität $\rightarrow$ Qualitätsinitiativen). Die Verbindung der Schweizer Ärztinnen und Ärzte hat damit eine Plattform für Wissensvermittlung und Vernetzung von Qualitätsbestrebungen in der Schweiz geschaffen, welche die Bandbreite der medizinischen Qualitätssicherung und -entwicklung in der Schweiz aufzeigt. Die Online-Plattform wird nun neu konzipiert und aktualisiert. Neben den ärztlichen Berufsgruppen wird neu auch den nicht-ärztlichen Berufsgruppen/Organisationen Raum geboten, um ihre Bestrebungen in der Qualität zu dokumentieren und zu veröffentlichen. Aufgenommen werden Qualitätsinitiativen, bei denen es sich um eine Massnahme zur Sicherung und Verbesserung der ärztlichen und medizinischen Leistung handelt. Zudem sollen die Qualitätsaktivitäten Prozesse verbessern und Fehler reduzieren, was sich positiv auf die Patientensicherheit und die Behandlung auswirken kann. Initianten von Q-Initiativen können Einzelpersonen, Netzwerke oder Institutionen sein, die nachweisbar Qualitätsentwicklung betreiben. Die Band-

Möchten Sie Ihre Qualitätsinitiative auf der Online-

Plattform der FMH publizieren?

Hier finden Sie den Link: http://www.fmh.ch/saqm/_service/

qualitaetsinitiativen.cfm

Kontaktieren Sie uns über ddq[at]fmh.ch oder 03135911 11, wir informieren

Sie gerne detailliert über das weitere Vorgehen.
Nutzen Sie die Gelegenheit und melden Sie Ihre Qualitätsaktivitäten für die Aufnahme auf der Online-Plattform für Qualitätsinitiativen in der Medizin der SAQM/FMH an!

breite der Q-Initiativen reicht von Kleinprojekten bis hin zu etablierten Qualitätsmanagementsystemen.

\section{Per Suchmaschine mit wenigen Klicks beim gesuchten Projekt}

Die Online-Porträts der vorgestellten Qualitätsinitiativen enthalten einen Kurzbeschrieb sowie weitere Informationen wie Ablauf, Zeitaufwand, Kosten und Kontaktdaten. Mit einer gezielten Suche nach Fach, Arbeitsbereich und Sparte lassen sich rasch all jene Qualitätsinitiativen finden, die den Bedürfnissen entsprechen.

\section{Aufnahme von neuen Qualitätsinitiativen}

Nach wie vor gibt es viele Qualitätsinitiativen, die noch nicht auf der FMH-Website präsent sind und einem breiten Fachpublikum vorgestellt werden sollten. Nutzen Sie die Gelegenheit und melden Sie Ihre Qualitätsaktivitäten für die Aufnahme auf der Online-Plattform «Qualitätsinitiativen SAQM» an.

Alle neuen Q-Initiativen, die bei der Abteilung Daten, Demographie und Qualität DDQ der FMH eingegangen sind, beurteilt der Steuerungsausschuss der SAQM anhand von Kriterien zu Durchführbarkeit, Übertragbarkeit und zum Nutzen. Unter diesem Link können Sie die Angaben zu Ihrer Qualitätsinitiative direkt eingeben: http://www.fmh.ch/saqm/_service/qualitaetsinitiativen. $\mathrm{cfm}$. Weitere Informationen über die Aufnahme von Q-Initiativen finden Sie direkt auf unserer Website. 